\title{
Int3D: a data reduction software for single crystal neutron diffraction
}

Nebil A. Katcho, Laura Cañadillas-Delgado, Oscar Fabelo, Juan Rodríguez Carvajal 


\section{Why Int3D?}

\section{Status of the software in the single crystal diffractometers of the ILL $(D 9, D 10, D 19)$}

- Outdated software (dates from the 80 s, Fortran77, inefficient...)

- Software split in multiple pieces

- Difficult to deal with complex problems (twins, inconmensurability)

- Very limited and low quality data visualization

- Demands high expertise (high workload for local contacts) 


\section{The Int3D software}

- Integrates in a single application all the required tasks to perform the data reduction:

$>$ Peaks search

$>$ Determination of the orientation matrix

$>$ Integration of the reflections intensity

$>$ Parameters refinement (sample, instrument)

- It provides a graphical user interface that allows users:

Run crystallographic calculations

$>$ Visualize and interact with the data 


\section{Int3D: languages and libraries}

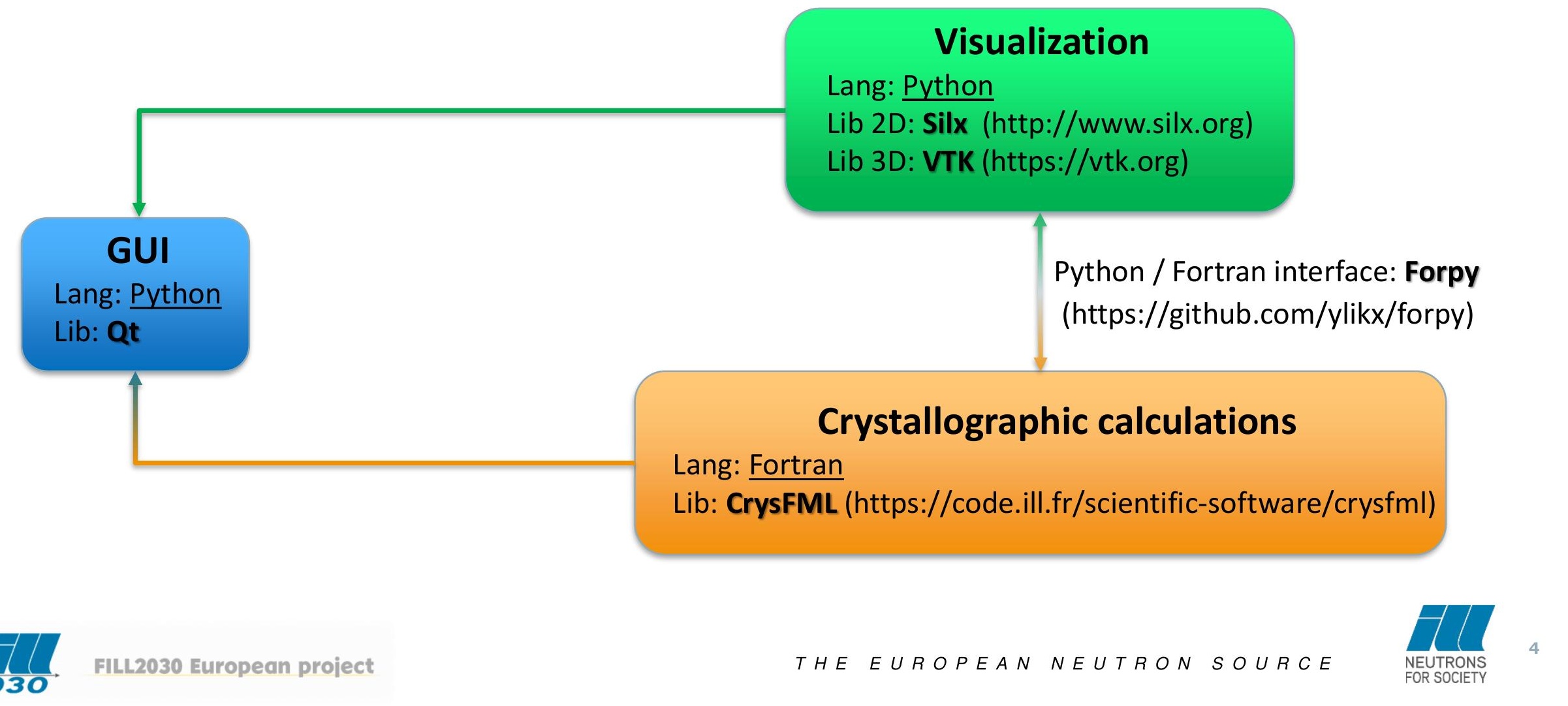




\section{Int3D: current status}

\begin{tabular}{|l|l|}
\multicolumn{1}{|c|}{ Instrument } & \multicolumn{1}{c|}{ Status } \\
\hline D9 & Ongoing, ready for next ILL cycle, January 2021 \\
\hline D10 & Not started yet, but straightforward extension from D9 \\
\hline D19 & Fully developed, ready to use \\
\hline
\end{tabular}




\section{D19 single crystal diffractometer}

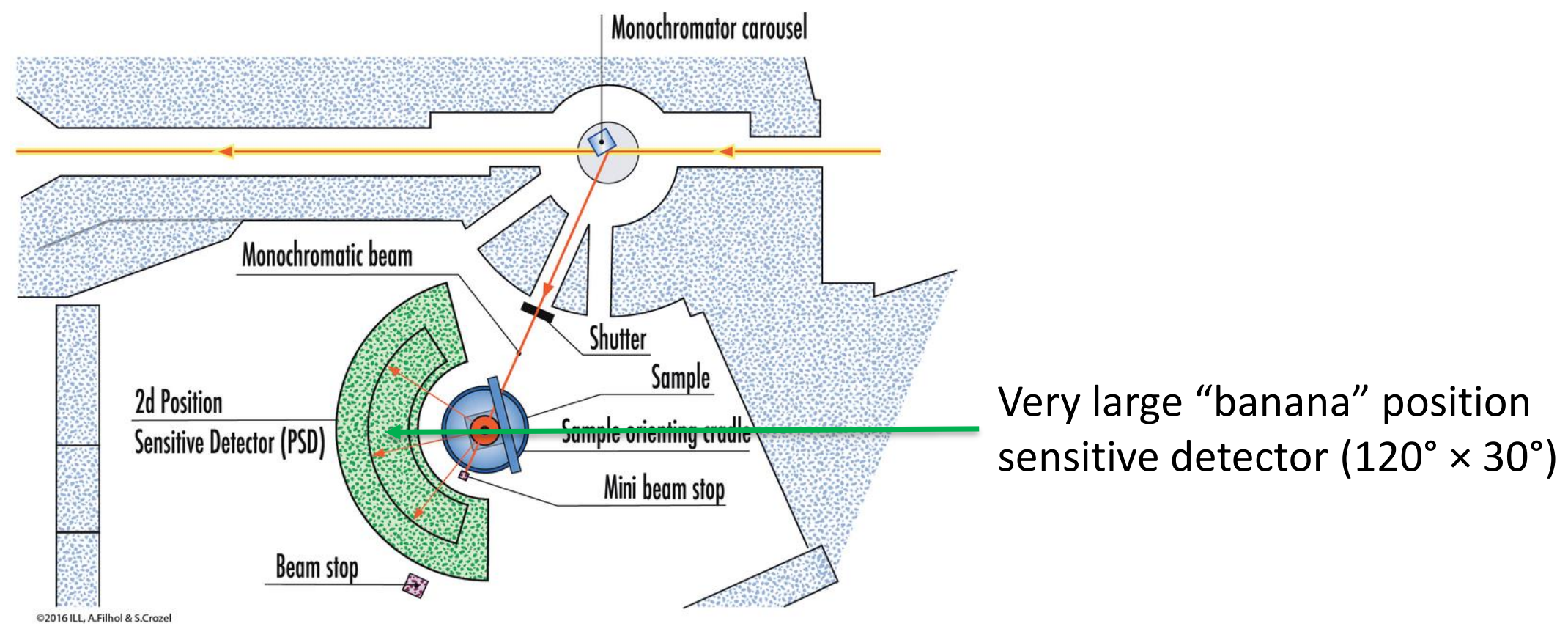




\section{Int3D: the GUI}

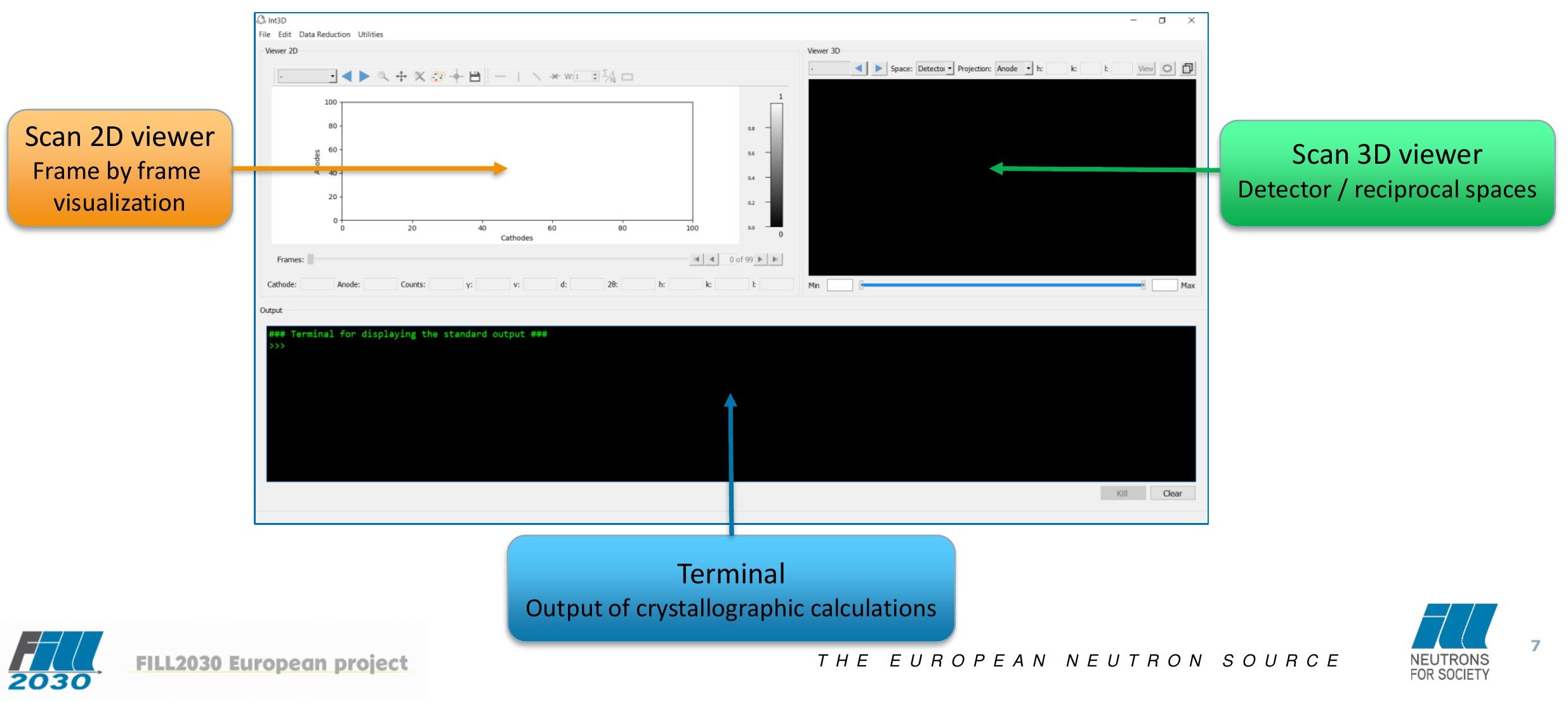




\section{Int3D: the raw data}

- The raw data for Int3D consists of a series of nexus files, called numors.

- Every numor corresponds to a given scan, usually an omega scan.

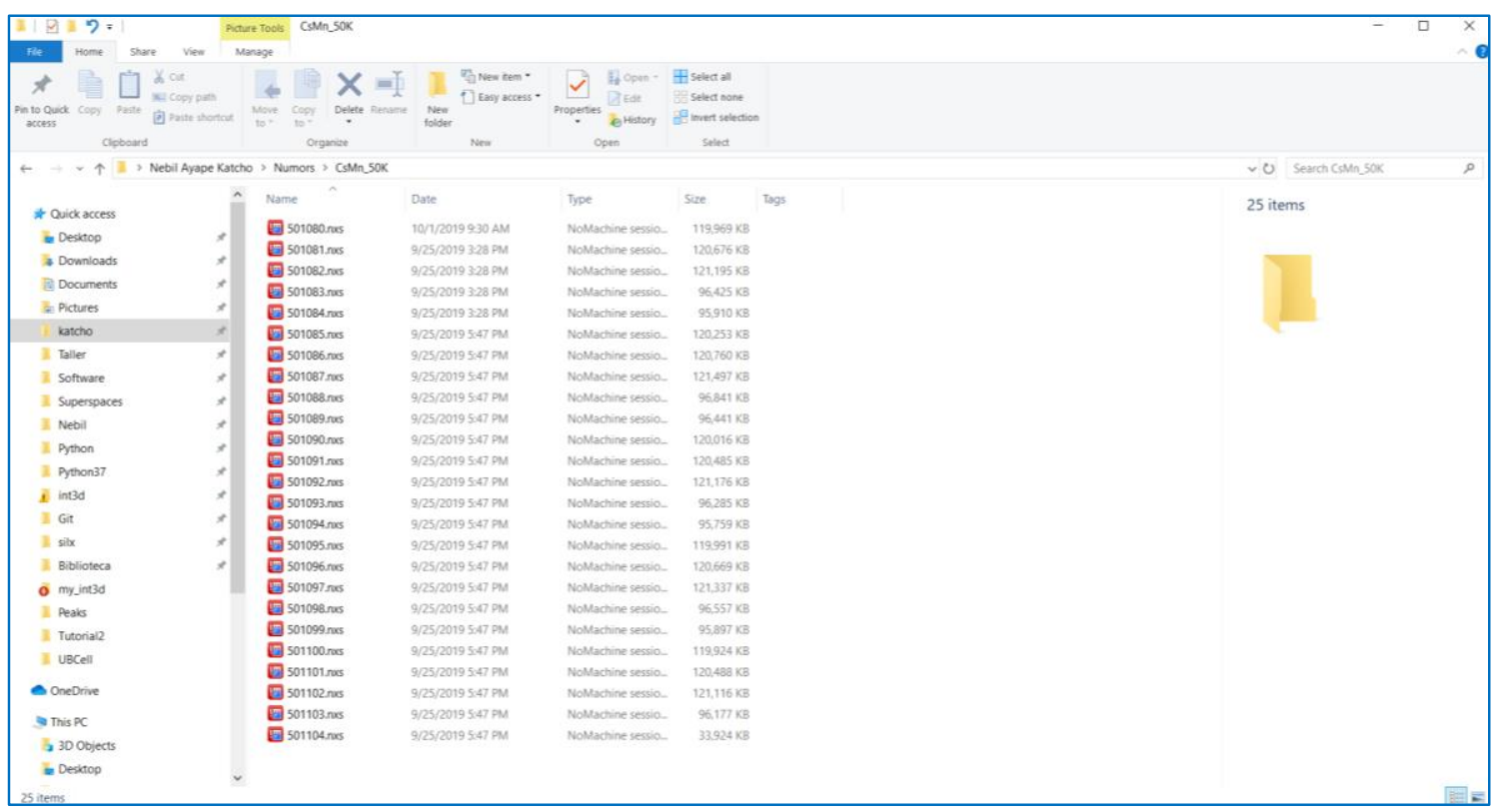




\section{Data reduction step by step}

1. Create a project and configure the instrument

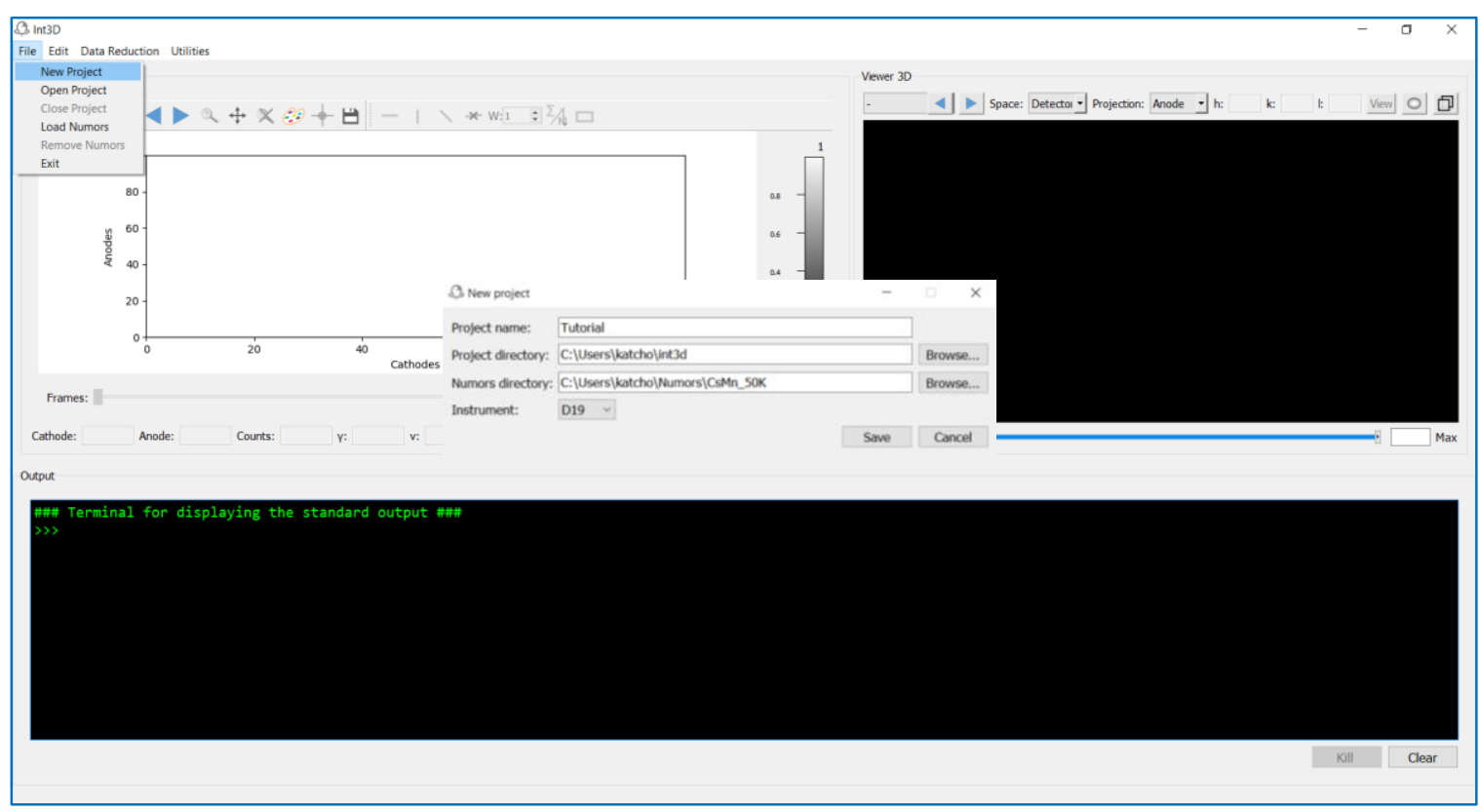




\section{Data reduction step by step}

1. Create a project and configure the instrument
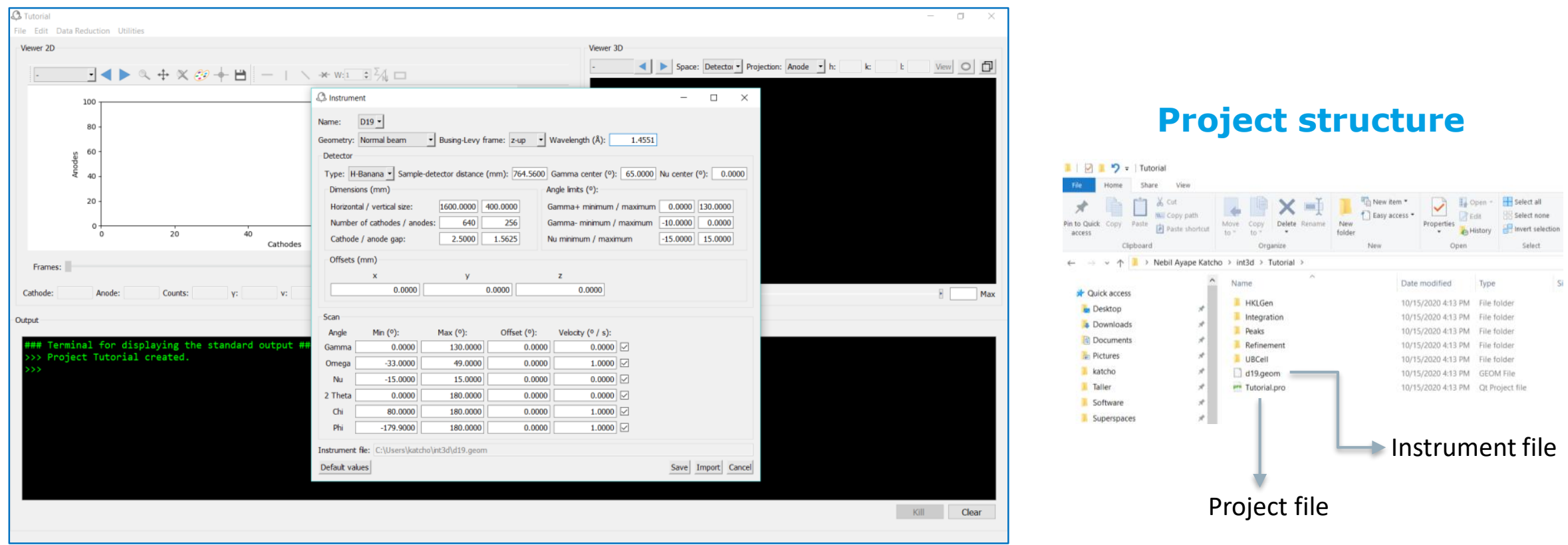


\section{Data reduction step by step}

\section{Appearance of D19 data}

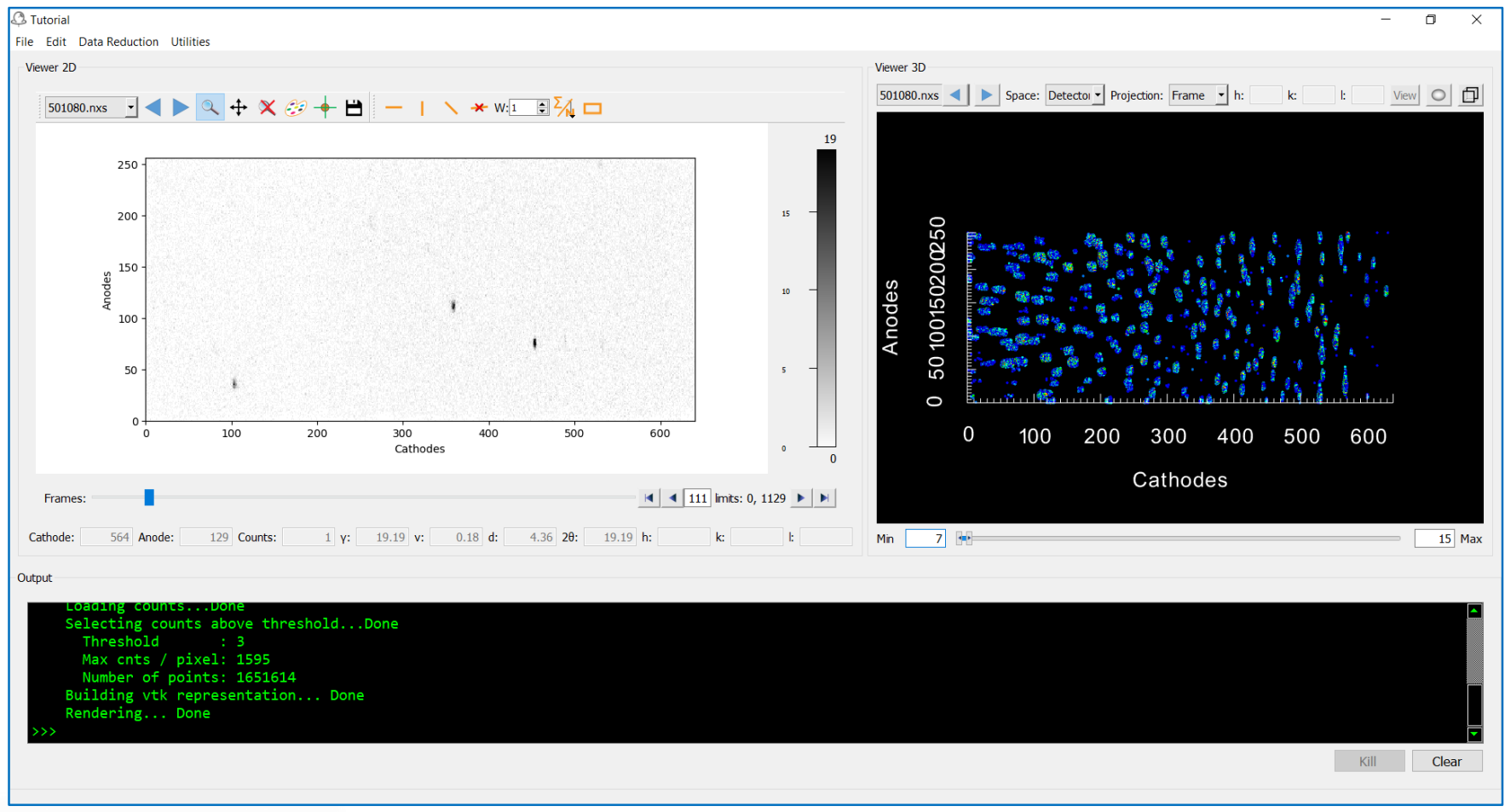




\section{Data reduction step by step}

\section{Peaks search}

From Data Reduction menu, open the Peakfind program

\section{Input}

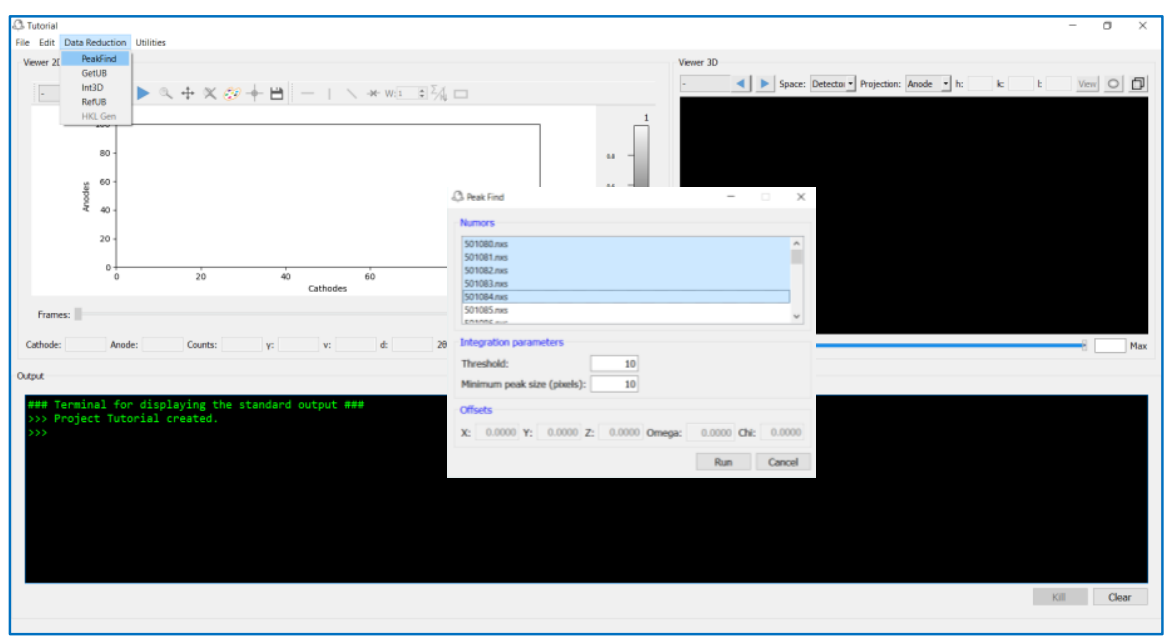

Output

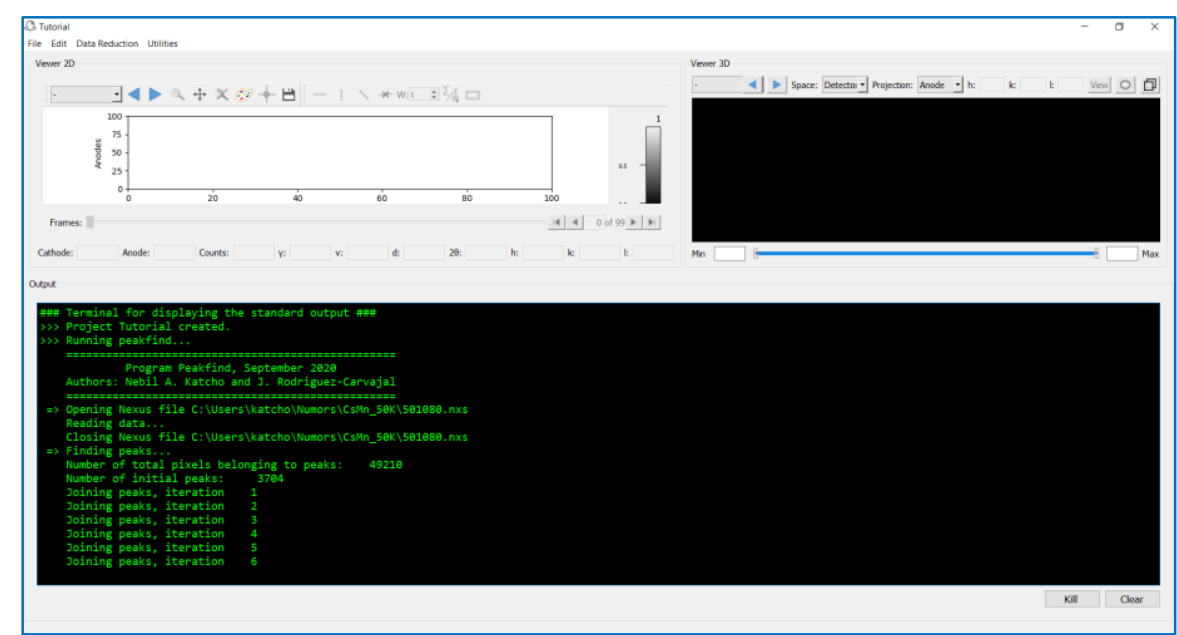

the european neutron source

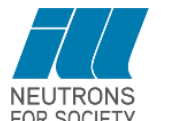




\section{Data reduction step by step}

\section{Determine the orientation matrix}

From Data Reduction menu, open the GetUB application

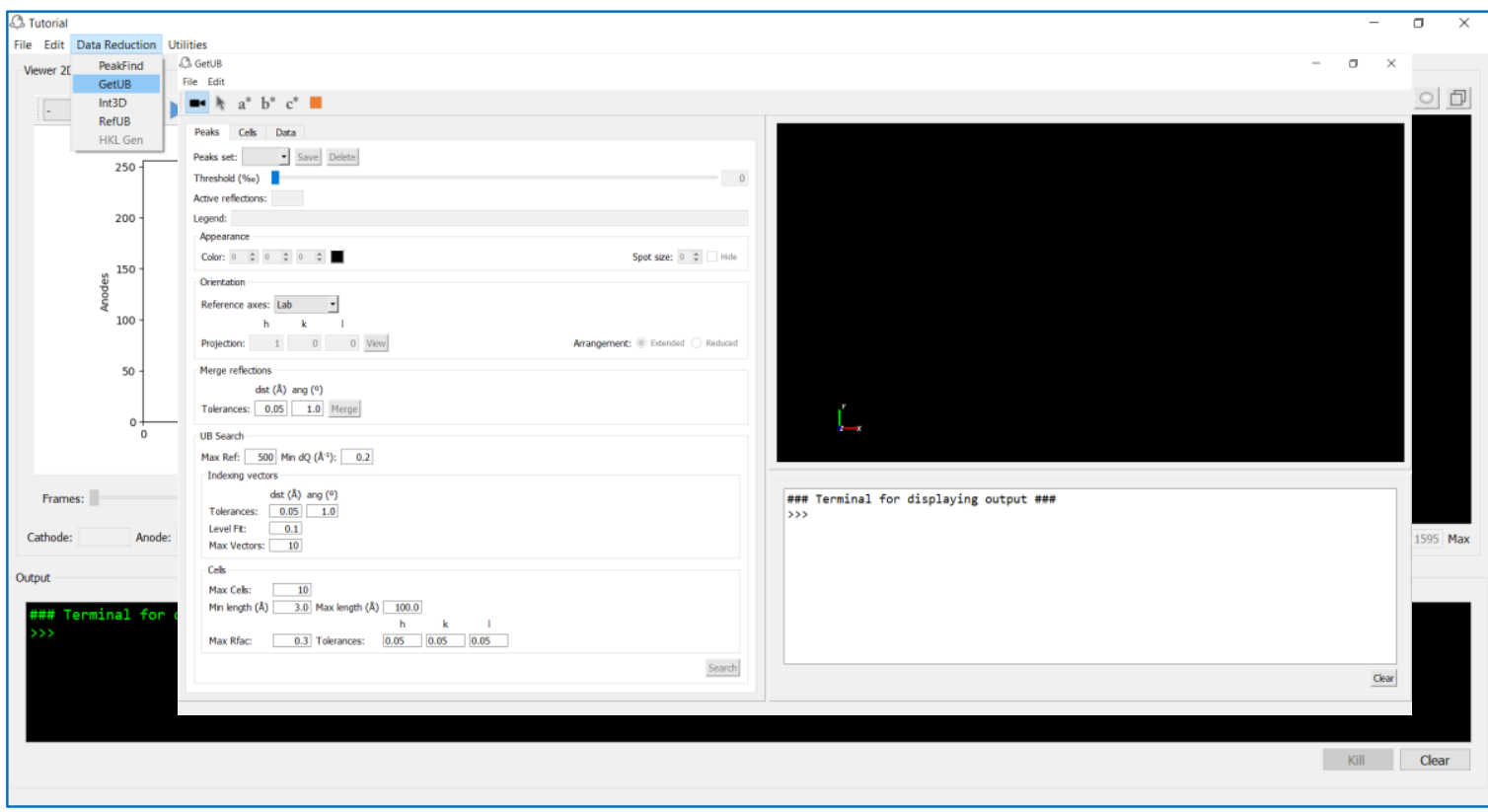

\section{GetUB}

- Determination of the orientation matrix

- Visualization of reciprocal space

- Orientation

- Interaction with the data:

- Classification

- Filtering

- Deletion 


\section{Data reduction step by step}

2. Determine the orientation matrix

Load peaks found by peakfind and search the UB matrix

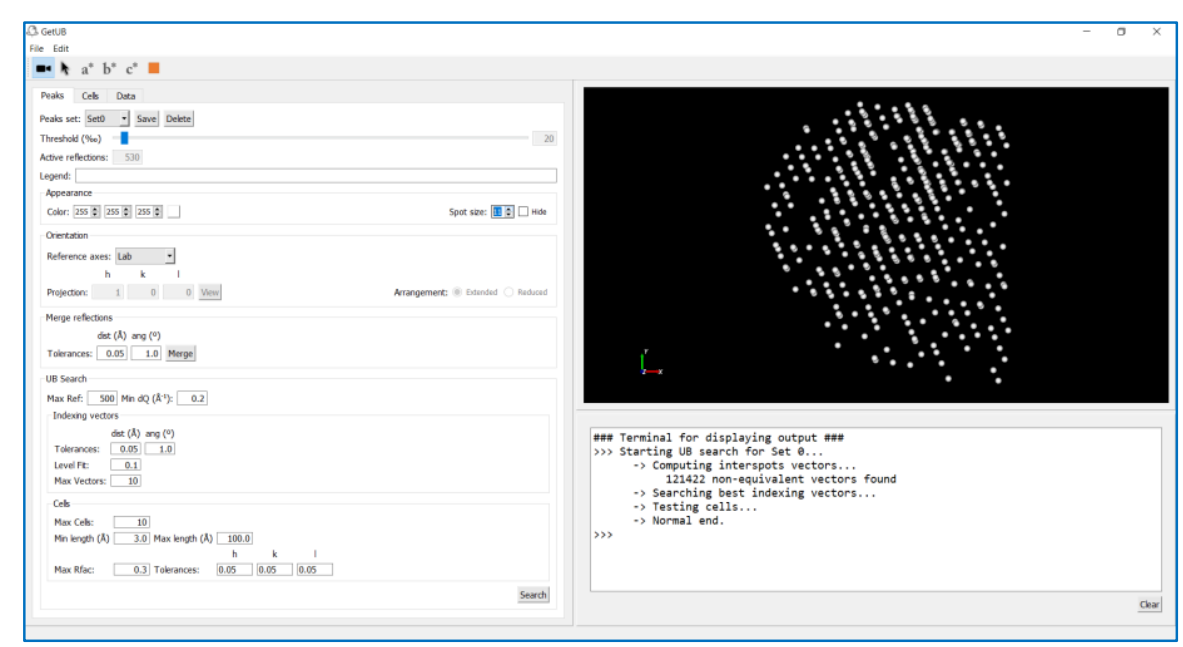

Check proposed cells

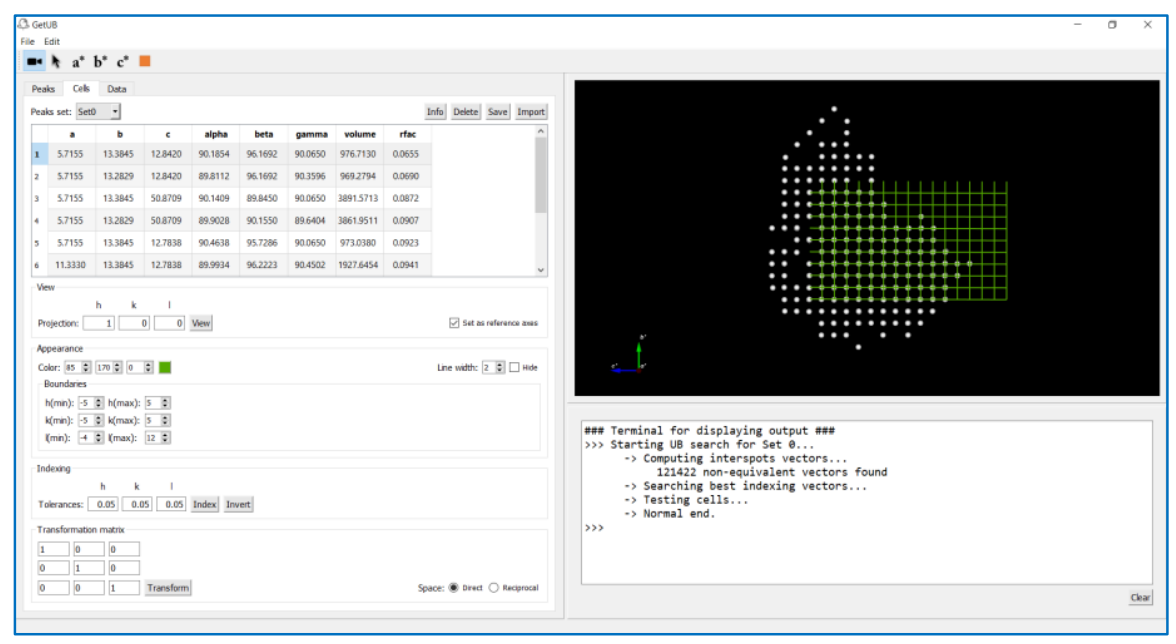

THE EUROPEAN NEUTRON SOURCE 


\section{Data reduction step by step}

\section{Load the UB matrix}

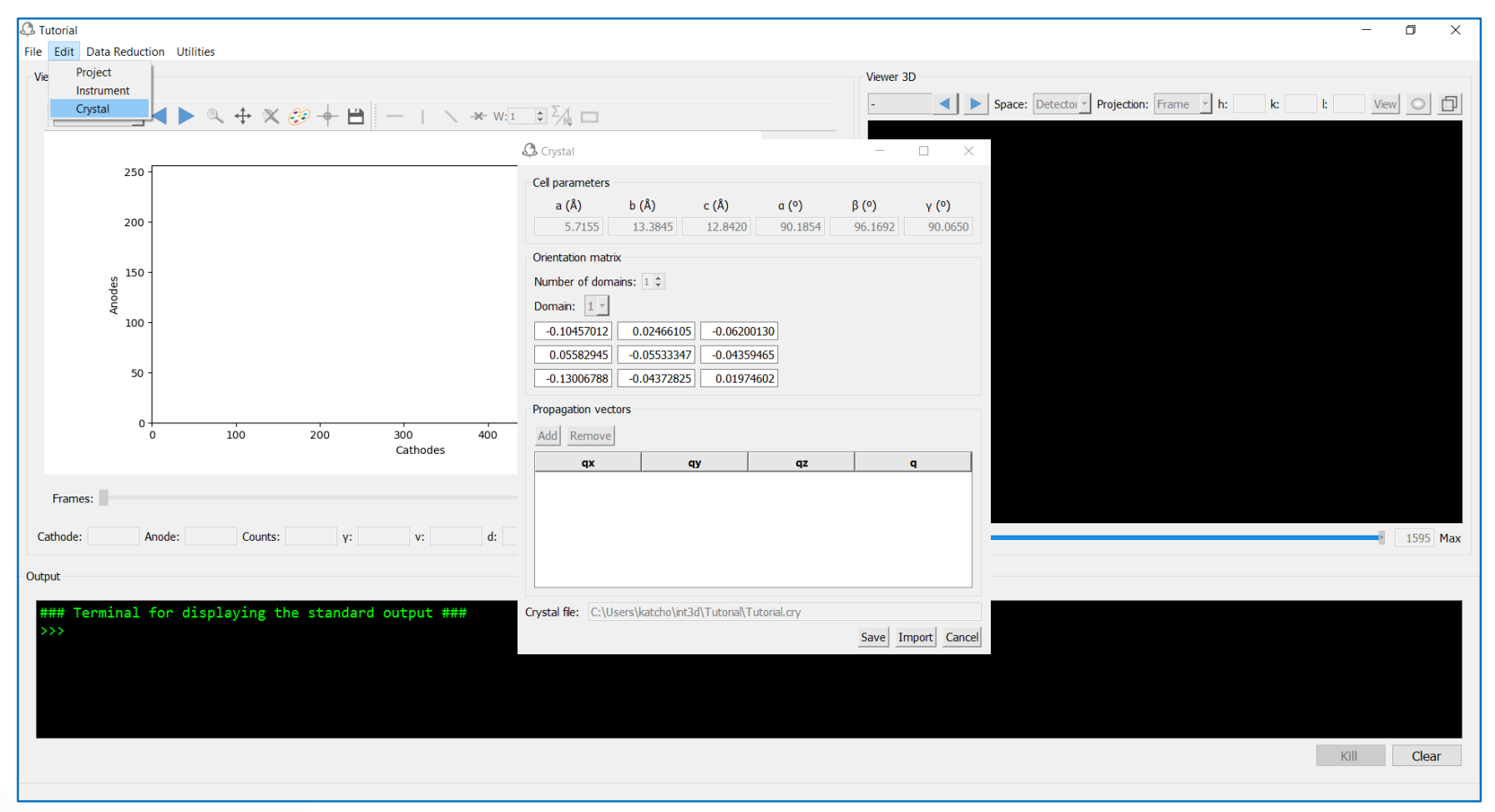




\section{Data reduction step by step}

Get the data indexed

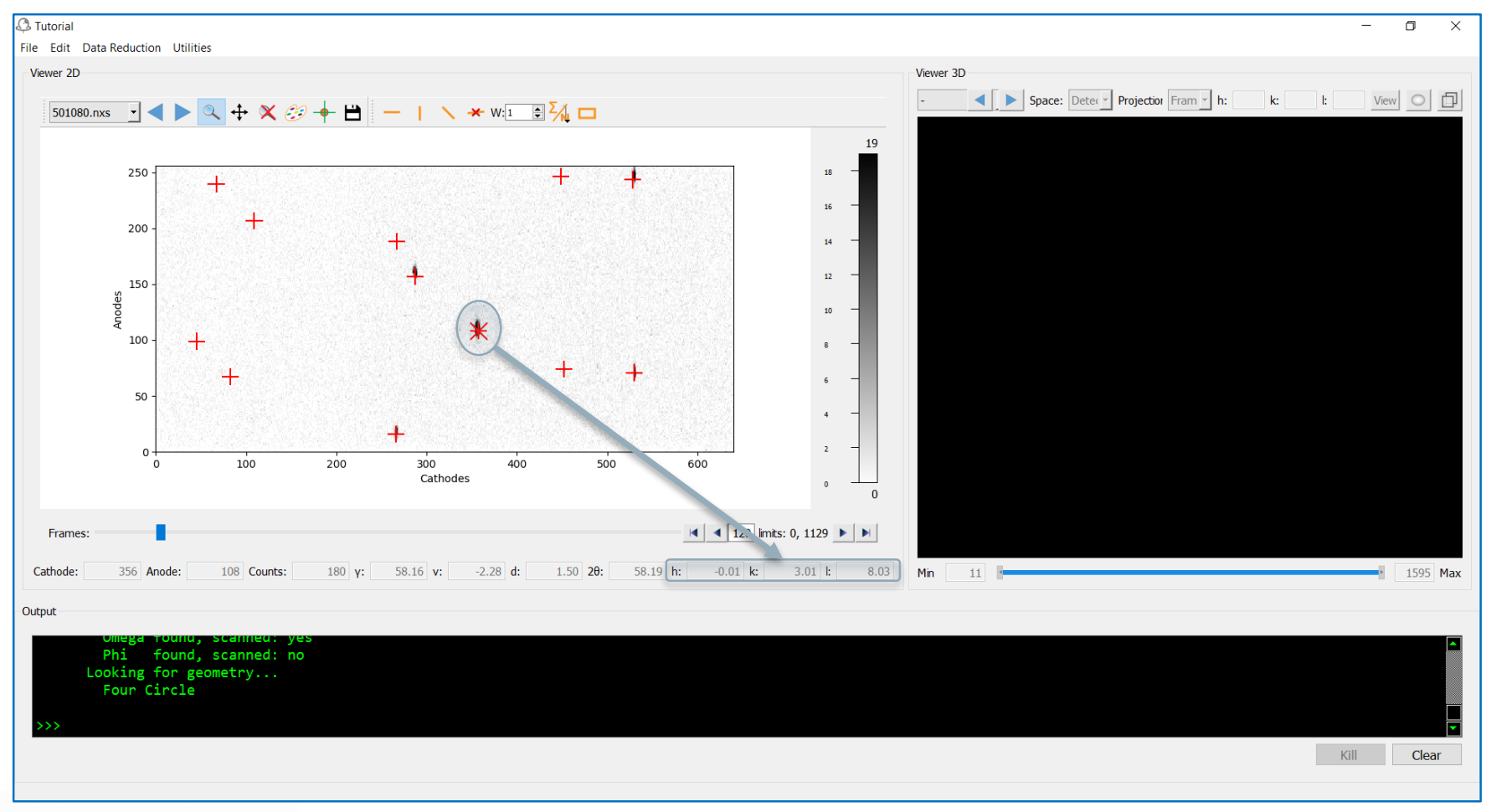




\section{Data reduction step by step}

\section{Integration}

From Data Reduction menu, open Int3D

\section{Input}

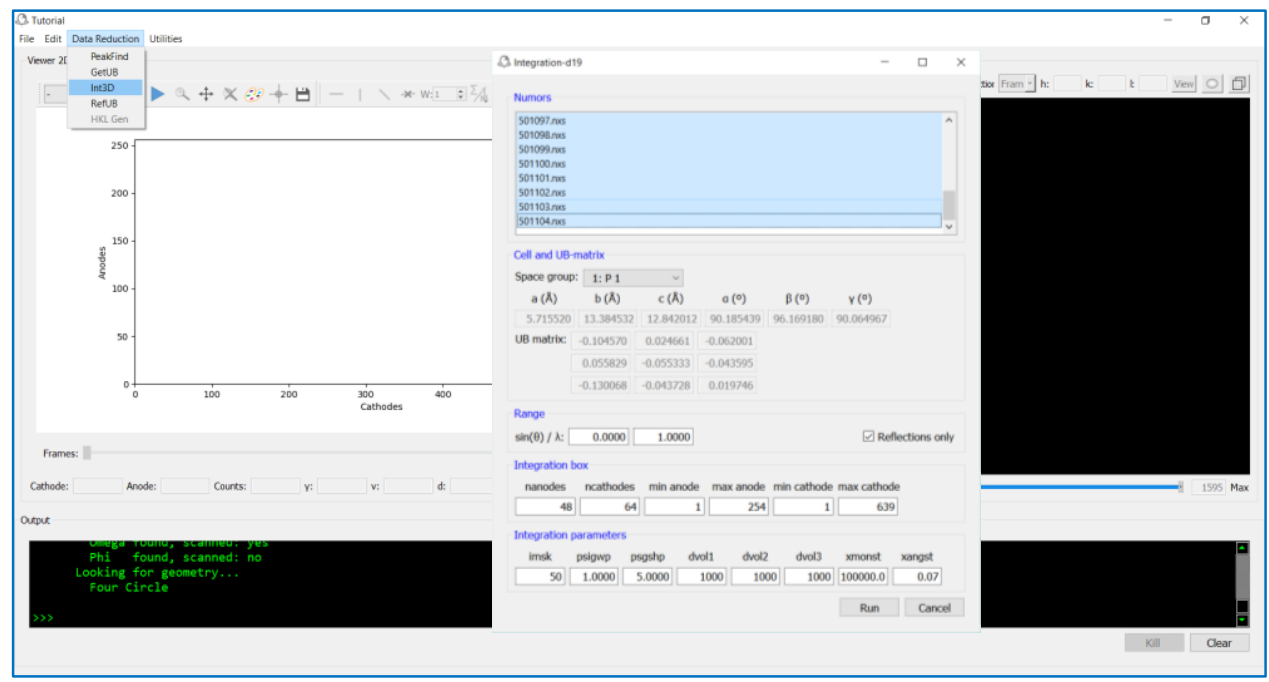

Output

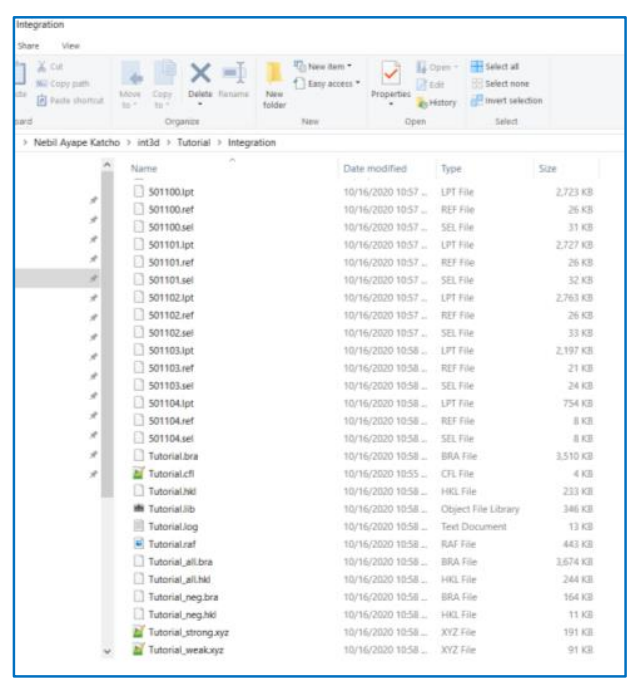

Files

.lpt : integration details ref : peak centroids sel : integration ellipsoids .hkl : integrated intensities lib : library for weak refl .raf : used for refinement 


\section{Data reduction step by step}

Check integration ellipsoids by visual inspection

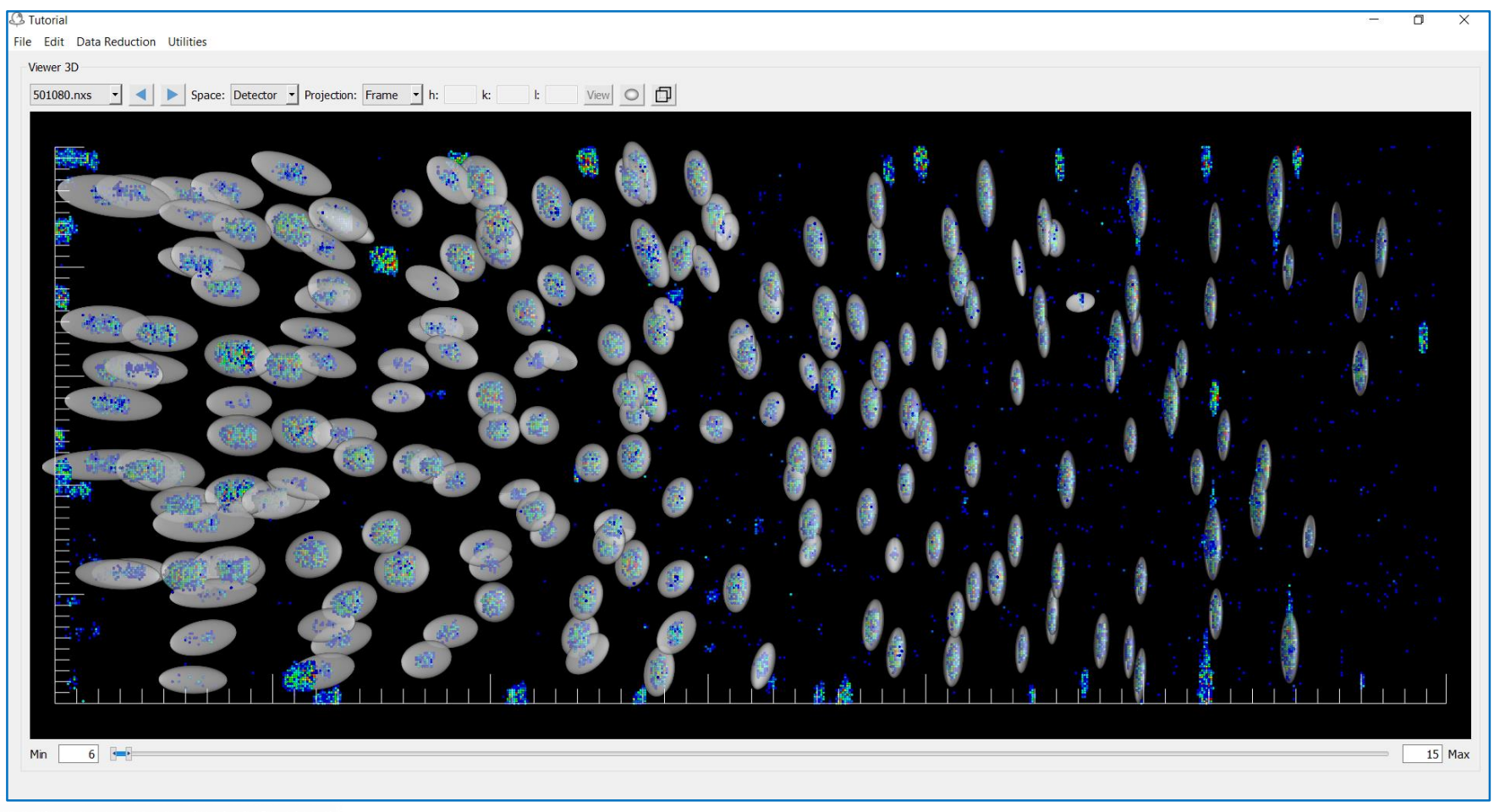




\title{
Data reduction step by step
}

\section{Check offsets}

Use GetUB to translate all reflections to the origin

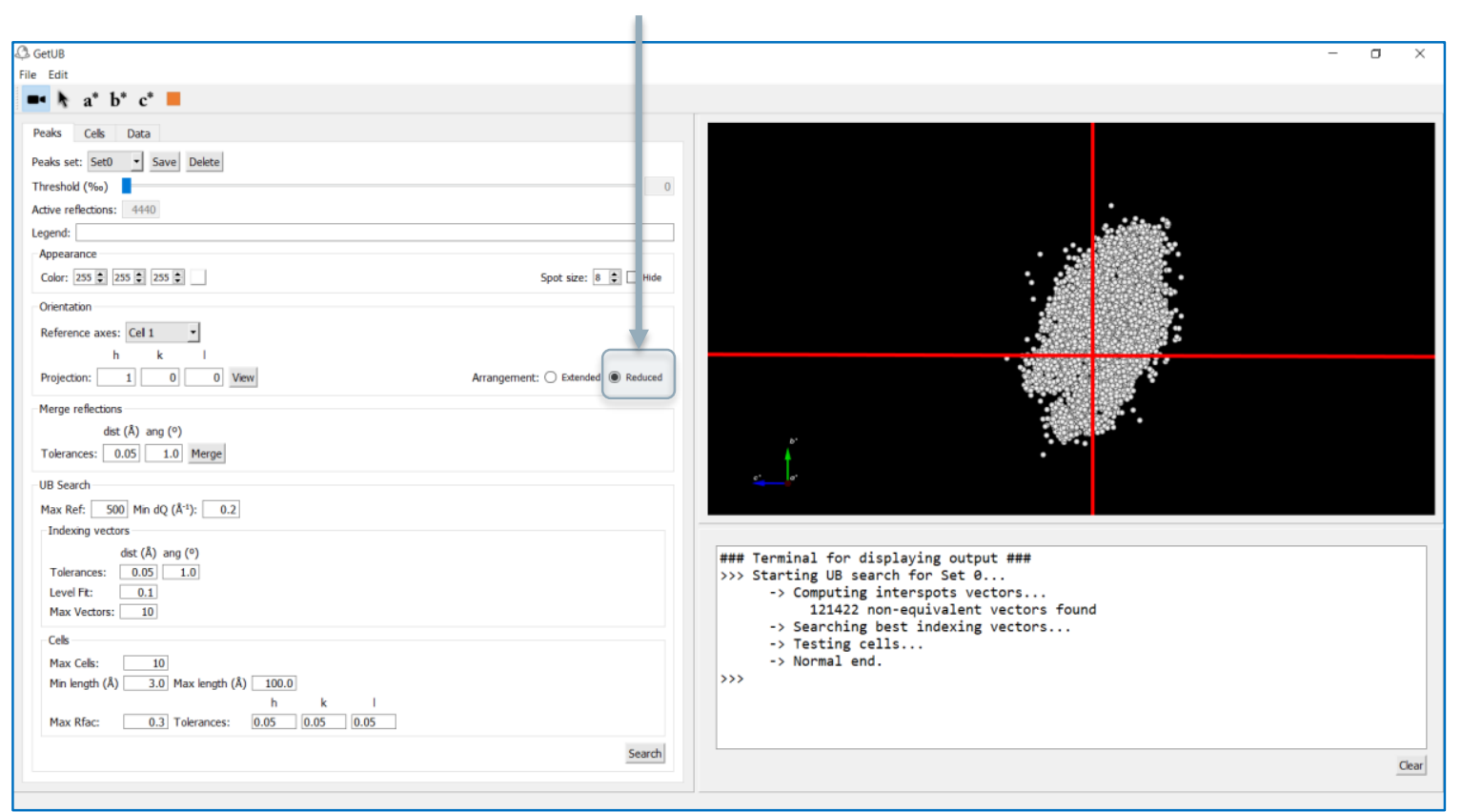

\author{
Not well centred \\ Anisotropy
}

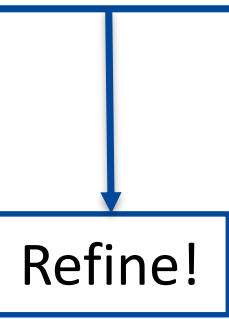




\section{Data reduction step by step}

\section{Refinement}

From Data Reduction menu, open RefUB

\section{Input}

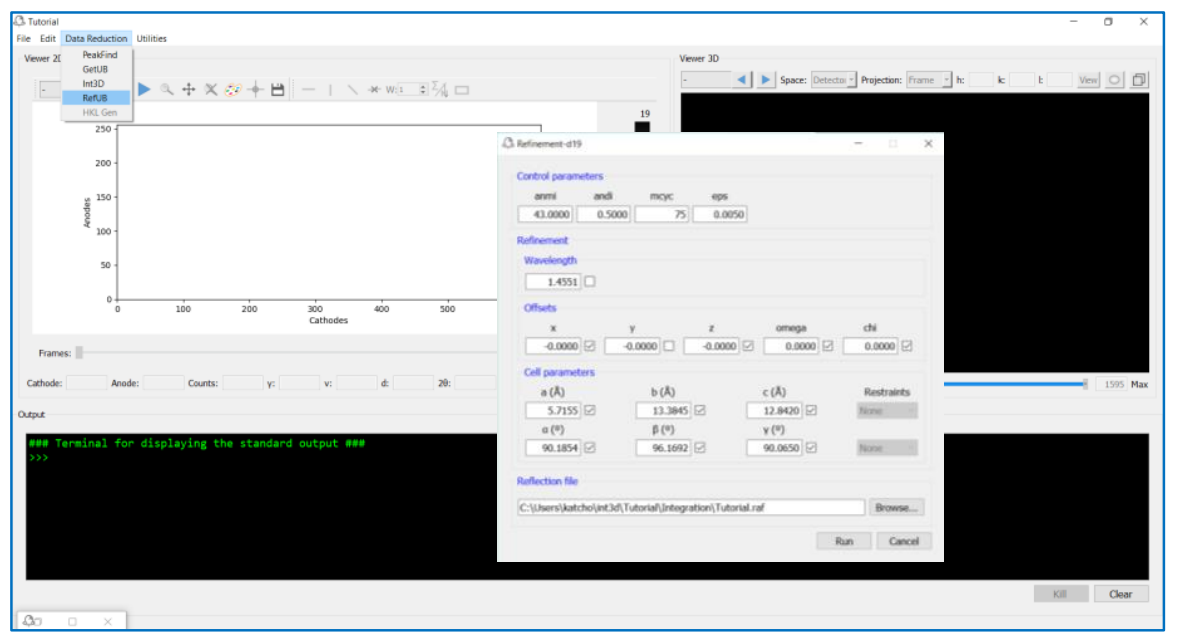

Output

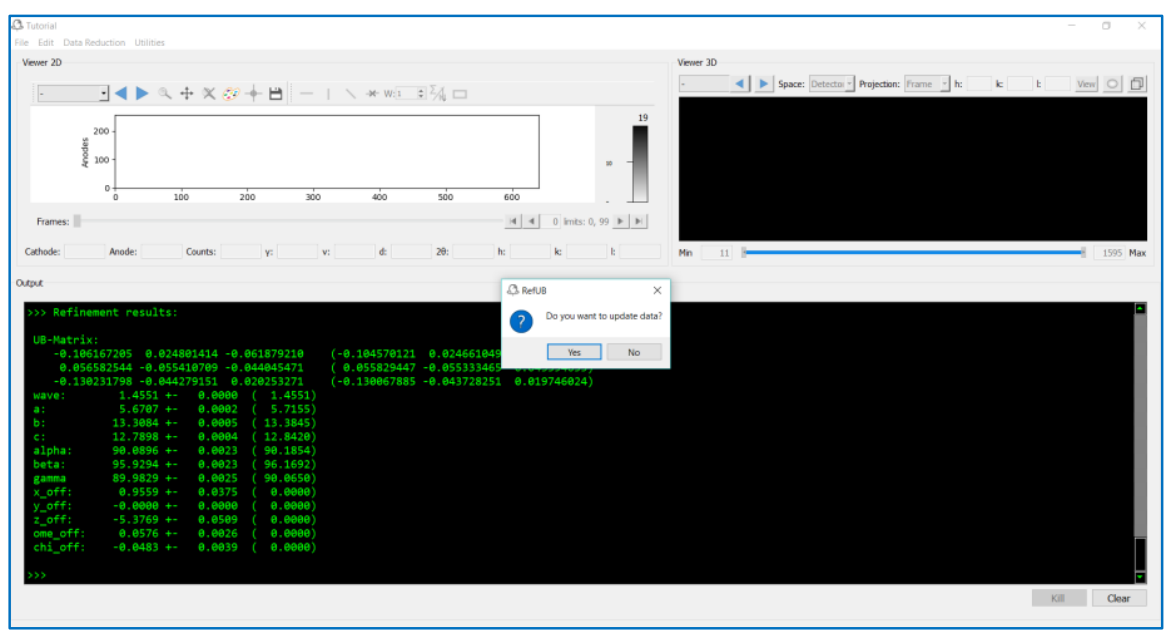

THE EUROPEAN NEUTRON SOURCE 


\section{Data reduction step by step}

\section{Check offsets}

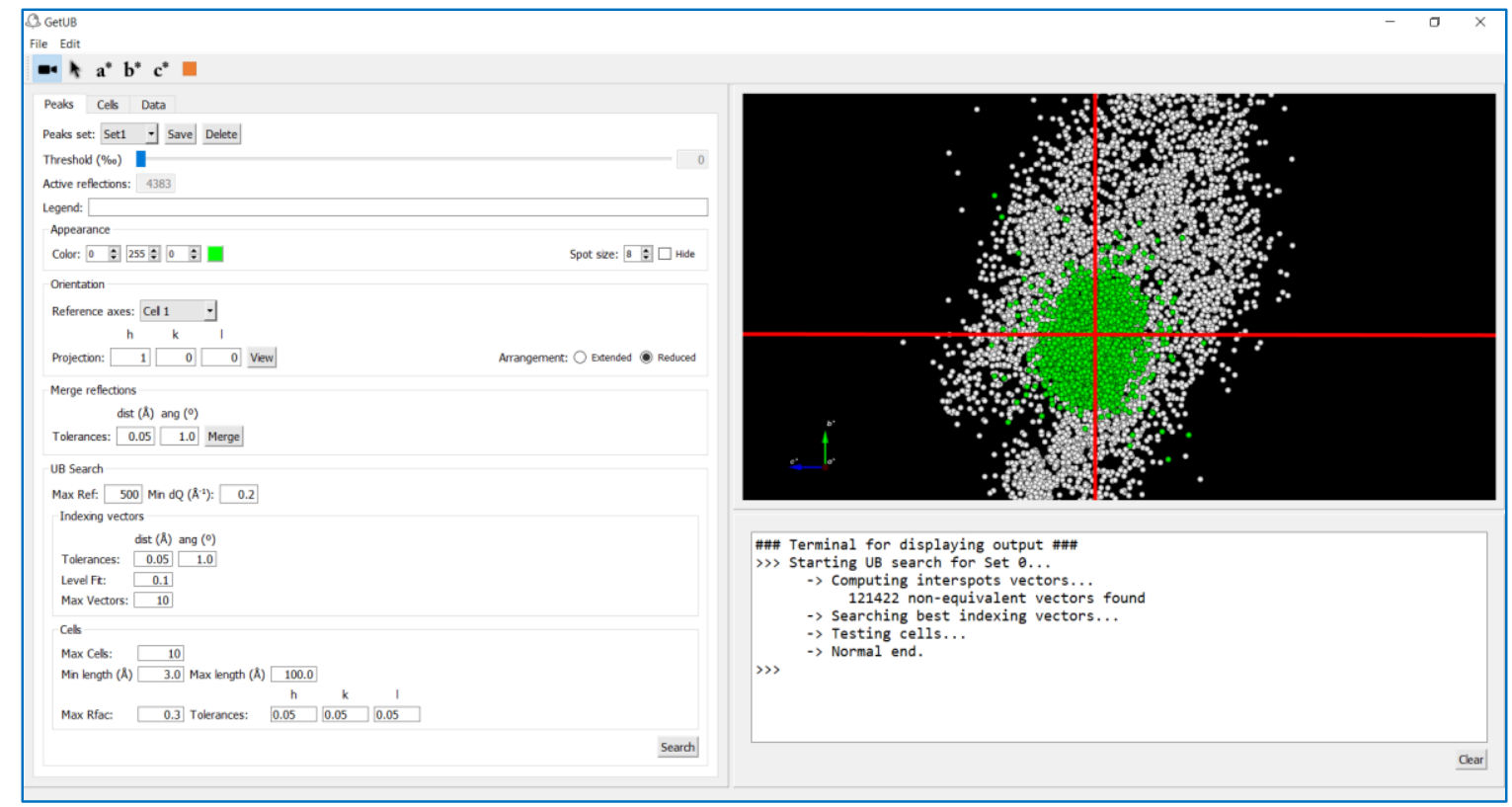

\begin{tabular}{|c|}
\hline Well centred \\
Isotropy \\
Less dispersion \\
\hline
\end{tabular}




\section{Summary and conclusions}

- We have integrated in a single application all the required tasks for performing the data reduction in single crystal neutron diffraction experiments.

- Int3D provides powerful graphical tools and suggest reasonable parameters for the different steps of the data reduction process, which makes it accessible to users without demanding high expertise.

- The application is distributed as a single folder containing all required libraries and executables. No installation of libraries or python is required.

- Video tutorial: www.int3d.fr

- Future improvements: absorption correction, complete visualization of data in reciprocal space, users demands... 
